Please do not remove this page

RMIT

UNIVERSITY

\title{
The directivity of the sound radiation from panels and openings
}

Davy, John

https://researchrepository.rmit.edu.au/esploro/outputs/9921864184301341/filesAndLinks?institution=61RMIT_INST\&index=null

Davy, J. (2009). The directivity of the sound radiation from panels and openings. Journal of the Acoustical Society of America, 125(6), 3795-3805. https://doi.org/10.1121/1.3117687

Document Version: Accepted Manuscript

Published Version: https://doi.org/10.1121/1.3117687

Repository homepage: https://researchrepository.rmit.edu.au

(c) 2009 Acoustical Society of America.

Downloaded On 2023/04/26 23:12:56 +1000

Please do not remove this page 
The directivity of the sound radiation from panels and openings ${ }^{\mathrm{a}}$

John L. Davy ${ }^{\text {bc }}$

School of Applied Sciences, RMIT University, GPO Box 2476V Melbourne, Victoria 3001, Australia

Running title: The directivity of panels and openings

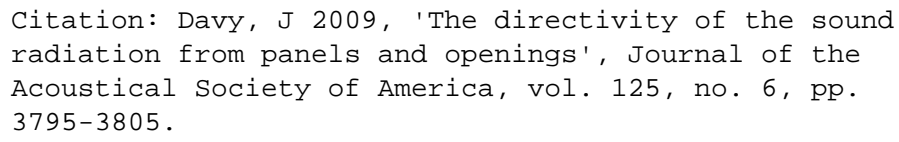

${ }^{a}$ Portions of this work were presented and published in series of conference papers and theses, by the author and his students, which are referred to in the introduction and references.

b Electronic mail: john.davy@,rmit.edu.au

${ }^{\mathrm{c}}$ Also employed at CSIRO Materials Science and Engineering, PO Box 56 Highett Victoria 3190, Australia, john.davy@csiro.au 
This paper presents a method for calculating the directivity of the radiation of sound from a panel or opening, whose vibration is forced by the incidence of sound from the other side. The directivity of the radiation depends on the angular distribution of the incident sound energy in the room or duct in whose wall or end the panel or opening occurs. The angular distribution of the incident sound energy is predicted using a model which depends on the sound absorption coefficient of the room or duct surfaces. If the sound source is situated in the room or duct, the sound absorption coefficient model is used in conjunction with a model for the directivity of the sound source. For angles of radiation approaching $90^{\circ}$ to the normal to the panel or opening, the effect of the diffraction by the panel or opening, or by the finite baffle in which the panel or opening is mounted, is included. A simple empirical model is developed to predict the diffraction of sound into the shadow zone when the angle of radiation is greater than $90^{\circ}$ to the normal to the panel or opening. The method is compared with published experimental results.

PACS numbers: 43.55.Rg, 43.50.Jh, 43.55.Ti, 43.50.Gf 


\section{INTRODUCTION}

This paper describes a method for predicting the directivity of the sound radiated from a panel or opening excited by sound incident on the other side. This directivity needs to be known when predicting the sound level at a particular position due to the sound radiation from a factory roof, wall, ventilating duct or chimney flue. The dependence on angle of incidence of the sound insulation of windows, doors, walls and roofs also needs to be known in order to predict the internal noise due to an external noise source.

The method described in this paper is mainly two dimensional. The directivity is modelled as being that of a line source whose phase of vibration is determined by the angle of incidence of the incident sound. The angular distribution of the incident sound energy is a function of the sound absorption coefficient of the walls of the room or the duct, and the directivity of the sound source. The directivity of the sound source is modelled as being that of a line source of constant phase. The diffraction caused by the finite size of the opening or panel, and any baffle that it is mounted in, is also modelled. An empirical model is used to predict the diffraction of sound into the shadow zone. In some cases a correction is made for the effects of "background" scattering.

A number of authors report experimental measurements of the directivity of openings and panels in the external wall of a room. Rindel (1975) measures the directivity of openings and single and double leaf panels mounted in the wall of a scale model room mounted in an anechoic room. His measurements are from outside to inside the scale model room. It should be noted that Rindel measures and removes the sound pressure increase on the surface of the panel due to diffraction from his experimental results. The diffraction theory developed in this paper is based partly on Rindel's measurements of the sound pressure increase on a panel due to diffraction. Oldham and Shen (1982) make anechoic room measurements of the directivity of openings in the 
wall of a scale model room in the opposite direction. Roberts (1983) conducts measurements of the directivity of openings in the wall of a room from inside to outdoors. Shen and Oldham (1982) and Oldham and Shen (1983) extend their earlier anechoic room measurements to the directivity of single panels in the wall of their scale model room. Rowell and Oldham (1996) carry out near field acoustical holography measurements of the directivity of homogeneous, profiled and composite single leaf panels in the wall of a room. Stead (2001) measures the directivity of a single panel in the wall of a room from outdoors to inside.

In addition to experimental measurements, a number of theoretical and numerical models for predicting the directivity of panels and openings in the external wall of a room are also reported in the literature. Rindel (1975) develops a theory for the directivity of openings and panels in the wall of a room. Oldham and Shen (1982) develop a computer model with experimentally determined corrections to predict the directivity of an opening in a room wall. Shen and Oldham (1982) present a numerical model for the directivity of a panel in the wall of a room. Oldham and Shen (1983) develop an empirical model for the directivity of a panel in the wall of a room from their experimental data. The model presented in this paper is primarily a theoretical analytic model, although it does include some empirical equations and does involve one linear integral which needs to be integrated numerically. It is an extension of Rindel's theory, but is different from Shen and Oldham's theories. The model in this paper extends Rindel's theory in a number of ways. It uses an analytic model for the radiation efficiency rather than an interpolation from a table of numerically calculated values. An empirical analytic model for the sound pressure increase on the surface of a panel due to diffraction replaces Rindel's measurements. A theoretical model for the directivity of the sound which excites the panel replaces Rindel's assumption of a reverberant field. 
In the case of the opening at the end of a duct, a number of authors have published measurements and prediction methods for the directivity. Wells and Crocker (1953) make measurements on models and a prototype of an exhaust stack. Waters et al. (1955) and Björk (1994) conduct similar measurements on full scale and model exhaust stacks. Beranek (1954) presents measured directivity patterns for the inlet of a ventilating fan, while Allen (1960), Sharland (1972), and Woods (1972) give graphs or tables for estimating the directivity of duct openings in rooms.

Bies and Hansen (2003) give directivity curves for the radiation of sound from the end of a duct based on measurements in an anechoic room by Croft (1979) and Sutton (1990). These anechoic room measurements are continued by Dewhirst (2002) and Li (2005). Hansen supplied Dewhirst's and Li's data to the author of this paper in machine readable form. Neish (1997) and Potente et al. (2006) measure the directivity of a duct end in the open air. Day supplied the author of this paper with all his test data in machine readable form, including some unpublished data.

The classical theoretical paper on the directivity of an unflanged circular duct end is Levine and Schwinger (1948). Unfortunately, because it assumes plane wave propagation in the duct, it over estimates the experimentally observed duct directivity and can only be used up to the cut on frequency of the first cross mode. Joseph and Morfey (1999) extend the theory into the cross mode region. Wells and Crocker (1953) also calculate the directivity of circular and square cross section duct ends assuming plane wave propagation.

In a series of conference papers and theses, the author of this paper and his students gradually develop the method described in this paper. The derivation of the necessary results on the radiation efficiency of finite size flat panels is given by Davy (2004). Pavasovic (2006) and 
Davy and Pavasovic (2006) present the first version of this model which assumes a cosine squared angular distribution of the incident sound energy. Davy and Kannanaikkel John (2006) replace this cosine squared distribution with a physical model for the angular distribution of the incident sound energy which depends upon the sound absorption coefficient of the side walls of the room or duct. They also introduce a simple line source directivity model for a sound source in a duct or room. Fisher (2006) compares the cosine squared and sound absorption coefficient models for the angular distribution of incident sound energy against the experimental directivity of openings and panels in the wall of a room. He introduces an automated method of determining the optimum value of the weighting angle which is used in the cosine squared model and an experimentally determined diffraction correction term. Fisher also shows that the models are not successful at predicting the directivity of cavity walls such as double glazing. Davy (2007) develops a model for predicting the diffraction on a finite flat surface as a function of angle of incidence and surface size. Davy (2007) and Davy and Kannanaikkel John (2007) introduce this diffraction model into the directivity model. This paper and Davy (2008) extend the diffraction model into the shadow zone and introduce a rule for calculating the average length of the line source which models the directivity of sound sources in ducts or rooms. The comparisons in this paper and Davy (2008) are based on a larger range of experimental results than the earlier papers. The purpose of this paper is to make the details of the directivity model easily and widely available.

\section{THEORY}

\section{A. Radiation efficiency and transmitted sound pressure}

The effective impedance $Z_{e}(\phi)$ of a finite panel in an infinite baffle to a plane sound wave incident at an angle of $\phi$ is (Rindel, 1975) 


$$
Z_{e}(\phi)=Z_{w f i}(\phi)+Z_{w f t}(\phi)+Z_{w p}(\phi)
$$

where

$Z_{w f i}(\phi)$ is the wave impedance of the fluid as experienced by the finite panel in an infinite baffle, whose vibration is due to a plane sound wave incident at an angle of $\phi$, on the side from which the plane sound wave is incident (this is the fluid loading on the incident side), $Z_{w f t}(\phi)$ is the wave impedance of the fluid as experienced by the finite panel in an infinite baffle, whose vibration is due to a plane sound wave incident at an angle of $\phi$, on the side opposite to which the sound is incident (this is the fluid loading on the non-incident or transmitted side) and $Z_{w p}(\phi)$ is the wave impedance of the finite panel in an infinite baffle to a plane sound wave incident at an angle of $\phi$, ignoring fluid loading.

It is assumed that the fluid wave impedances on both sides are the same and the imaginary part of the fluid wave impedance is ignored (Rindel, 1975). That is

$$
Z_{w f i}(\phi)=Z_{w f t}(\phi)=\rho c \sigma(\phi)
$$

where $\rho$ is the density of the fluid, $c$ is the speed of sound in the fluid and $\sigma(\phi)$ is the radiation efficiency into the fluid of one side of the finite panel in an infinite baffle, whose vibration is due to a plane sound wave incident at an angle of $\phi$.

Reflections at the panel edges are ignored (Rindel, 1975). The normal velocity $v(\phi)$ of the panel due to a plane sound wave incident at an angle of $\phi$ which exerts a pressure $p_{i}(\phi)$ is

$$
v(\phi)=\frac{p_{i}(\phi)}{2 \rho c \sigma(\phi)+Z_{w p}(\phi)} .
$$


The transmitted sound pressure $p_{t}(\theta, \phi)$ which is radiated by the panel on the non-incident side to a receiving point which is at an angle of $\theta$ to the normal to the centre of the panel and a large distance from the panel (see figure 1) is (Davy, 2004)

$$
p_{t}(\theta, \phi) \propto v(\phi) \frac{\sin [k a(\sin \theta-\sin \phi)]}{k a(\sin \theta-\sin \phi)}
$$

where $k$ is the wave number of the sound and $2 a$ is the average length across the panel or opening in the plane containing the receiver and the normal to the panel or opening. Thus

$$
p_{t}(\theta, \phi) \propto \frac{p_{i}(\phi)}{2 \rho c \sigma(\phi)+Z_{w p}(\phi)} \frac{\sin [k a(\sin \theta-\sin \phi)]}{k a(\sin \theta-\sin \phi)} .
$$

The case where the incident sound is generated by a sound source in a room or duct is now considered. It is assumed that the sound pressure waves are incident at different angles $\phi$ with random phases and mean squared sound pressures which are proportional to a weighting function $w(\phi)$.

$$
\left|p_{i}(\phi)\right|^{2} \propto w(\phi) .
$$

The weighting function is to account for the fact that sound waves at grazing angles of incidence will have had to suffer more wall collisions and therefore be more attenuated before reaching the panel. The total mean square sound pressure $\left|p_{T}(\theta)\right|^{2}$ at the receiving point is

$$
\left|p_{T}(\theta)\right|^{2} \propto \int_{-\pi / 2}^{\pi / 2} \frac{w(\phi)}{2 \rho c \sigma(\phi)+\left.Z_{w p}(\phi)\right|^{2}}\left\{\frac{\sin [k a(\sin \theta-\sin \phi)]}{k a(\sin \theta-\sin \phi)}\right\}^{2} d \phi .
$$

The case when sound is incident from a source in a free field at an angle $\theta$ to the normal to the panel and the panel radiates at all angles $\phi$ into a room or duct is also of interest. In this case the weighting function $w(\phi)$ is to account for the fact that sound waves radiated at grazing 
angles will have had more wall collisions and therefore be more attenuated before reaching the receiving position which is assumed to be a reasonable distance from the panel or opening which is radiating the sound. In this second case, it is necessary to integrate over all angles of radiation $\phi$ because of the reverberant nature of the sound. For this case, the impedance terms in the integral are functions of $\theta$ rather than $\phi$ and can be taken outside the integral. However in this study both cases are calculated using the formula for the first case which is shown above. This is because both cases should be the same by the principle of reciprocity and it is not clear which form of the formula is more appropriate.

For large values of $k a$, the two cases of the formula are similar. If $k a$ is much greater than 1 , the function

$$
\left\{\frac{\sin [k a(\sin \theta-\sin \phi)]}{k a(\sin \theta-\sin \phi)}\right\}^{2}
$$

has a sharp maximum at $\phi=\theta$ and is symmetrical in both $\theta$ and $\phi$ about the point $\phi=\theta$. These facts are exploited by evaluating the impedance terms for the first case at $\phi=\theta$ and taking them outside the integral. This gives the formula for the second case.

To derive the angular weighting function, it is assumed that the sound source is a distance $b$ from the surface of the room containing the panel or opening and that the room width is $g$ in the plane containing the incident sound ray (see figure 2). If the sound ray is incident at an angle of $\phi$ to the normal to the panel or opening, it travels a minimum distance of $b \tan \phi$ parallel to wall containing the panel or opening before hitting the wall. The sound which travels this minimum distance hits the wall approximately

$$
n=\frac{b}{g} \tan \phi
$$


times before reaching the panel or opening, where $n$ is allowed to be a real number rather than an integer in order to give a smooth weighting function. If the sound absorption coefficient of the walls of the room is $\alpha$, the sound intensity incident at an angle of $\phi$ to the normal is proportional to

$$
w(\phi)=(1-\alpha)^{n}=(1-\alpha)^{\frac{b}{g} \tan |\phi|} .
$$

Equation (10) gives the weighting function $w(\phi)$. If $\alpha$ is zero, a uniform diffuse field will be obtained. If $\alpha$ is not known, the use of the empirical value $\alpha=0.05$ is suggested for hard walled rooms and ducts since this value gives reasonable agreement when the theory is compared to experiment. In a room or a straight unobstructed duct, $b$ should be set equal to the perpendicular distance of the source from the plane containing the opening or panel. For other ducts $b$ should be set equal to the perpendicular distance of the closest bend or obstruction to the duct opening. For the comparisons made with ducts in this paper, the duct sound sources were assumed to be at the opposite end of the duct to the duct opening. This was approximately true for all the ducts considered in this paper.

In this study the radiation efficiency of a panel or opening of length $2 a$ and width $2 d$ is used. It is approximated with the following equation (Davy, 2004).

$$
\sigma(\phi)=\left\{\begin{array}{cc}
\frac{1}{\frac{\pi}{2 k^{2} a d}+\cos \phi} & \text { if }|\phi| \leq \phi_{l} \\
\frac{1}{\frac{\pi}{2 k^{2} a d}+\frac{3 \cos \phi_{l}-\cos \phi}{2}} & \text { if } \phi_{l}<|\phi| \leq \frac{\pi}{2}
\end{array}\right.
$$

where 


$$
\phi_{l}=\left\{\begin{array}{cc}
0 & \text { if } k a \leq \frac{\pi}{2} \\
\arccos \left(\sqrt{\frac{\pi}{2 k a}}\right) & \text { if } k a>\frac{\pi}{2}
\end{array}\right.
$$

and $k$ is the wave number of the sound and $2 a$ is the length of the panel in the direction of the receiver. Note that in Davy (2004) the width is assumed to be equal to the length.

For an opening with no panel in an infinite baffle, $Z_{w p}(\phi)=0$. For a finite panel in an infinite baffle, the infinite panel result for $Z_{w p}(\phi)$ is used.

$$
Z_{w p}(\phi)=m \omega\left\{j\left[1-\left(\frac{\omega}{\omega_{c}}\right)^{2} \sin ^{4}(\phi)\right]+\eta\left(\frac{\omega}{\omega_{c}}\right)^{2} \sin ^{4}(\phi)\right\}
$$

where $m$ is the surface density (mass per unit area) of the panel, $\eta$ is the damping loss factor of the panel, $\omega_{c}$ is the angular critical frequency of the panel and $\omega$ is the angular frequency of the sound. This result is expected to be the correct result when averaged over frequency, because this approach gives the correct result for point impedances when averaged over frequency and position on a finite panel (Cremer and Heckl, 1973). However, comparisons between theory and experiment show that it does not work particularly well at and above the coincidence frequency.

The directivity of the sound source is also included when it is in the duct or room. The sound source is modelled as a line source of length $2 r$ where $r$ is the radius of the sound source. The directivity of the sound source is proportional to

$$
\left[\frac{\sin (k r \sin \phi)}{k r \sin \phi}\right]^{2}
$$

where $k$ is the wave number.

\section{B. Diffraction due to the finite size of the baffle}


To correctly model the directivity, it is necessary to include the effect of diffraction due to the finite size of the baffle in which the panel or opening is mounted, or the finite size of the panel or opening if it is not mounted in a baffle. $p(\theta)$ is the ratio of the increased sound pressure to the sound pressure without the baffle for an angle of incidence or radiation of $\theta$. The baffle is of length $2 L$ in the plane containing the receiver (or source) and the normal to the baffle and of width $2 W$ in the direction at right angles to the above mentioned plane. Note that in Davy (2007), the length and width of the baffle are assumed to be equal.

Two cases are considered. The first is when the sound is incident internally on a panel or an opening mounted in the wall of a room or the end of a duct over a range of angles of incidence due to a sound source operating inside the room or the duct. In this first case, the receiver is a large distance away at a specified radiation angle. The second case is when the sound is incident externally on a panel or an opening mounted in the wall of a room or the end of a duct at a specified angle of incidence from a sound source external to the room or the duct which is a large distance away. In this second case, the sound reaches a receiver inside the room or the duct after being radiated over a range of angles. By the principle of reciprocity, these two cases should give the same directivity as a function of the specified angle of radiation or the specified angle of incidence.

Because the panel or opening may be mounted in a baffle, such as the external wall of a building, the average effects of diffraction over the whole of the rigid surface or over an area near the centre of the rigid surface need to be known. The theoretical model is developed by considering experimental and theoretical results for the second case.

\section{High Frequencies}


Consider the case of an infinite plane wave travelling in an infinite half space bounded by an infinite rigid plane. There is a discontinuity in the sound pressure on the surface of the rigid plane as a function of the angle of incidence. The sound pressure on the bounding infinite plane is double the sound pressure of the incident wave except when the angle of incidence is $\pi / 2 \mathrm{rad}$. In this exceptional case, the sound pressure on the bounding infinite rigid plane is equal to the sound pressure of the incident wave.

What happens when the rigid plane is finite in size? To answer this question for the high frequency case when the finite rigid planes are relatively large in terms of the wavelength, the experimental results for diffraction for the second case in Rindel (1975) are analysed. For third octave bands with centre frequencies from $200 \mathrm{~Hz}$ to $20 \mathrm{kHz}$, Rindel gives level recorder plots of the sound pressure level of a flush mounted microphone at the centre of a finite rigid plane as a function of angle of incidence from slightly less than $-\pi / 2 \mathrm{rad}$ to slightly greater than $\pi / 2 \mathrm{rad}$. Rindel makes measurements with two sizes of finite rigid plane. The larger finite rigid plane measures $2 L=3800 \mathrm{~mm}$ in the plane of the angle incidence and $2 W=3100 \mathrm{~mm}$ at right angles to the plane of the angle of incidence. The smaller finite rigid plane is the wall of Rindel's experimental box and measures $2 L=1360 \mathrm{~mm}$ in the plane of the angle incidence and $2 \mathrm{~W}=$ $1110 \mathrm{~mm}$ at right angles to the plane of the angle of incidence.

Except for planes which are relatively small in terms of the wavelength, Rindel's experimental results are approximately constant from a frequency dependent negative angle of incidence $-\theta_{m}$ rad to a frequency dependent positive angle of incidence $\theta_{m}$ rad. Because the magnitudes of the experimental values of these two angles of incidence are not exactly equal, their two magnitudes are averaged to give an experimental estimate of the frequency dependent magnitude $\theta_{m}$ rad. For planes which are relatively small in terms of the wavelength, $-\theta_{m}\left(\theta_{m}\right) \mathrm{rad}$ 
is measured as the angle of incidence closest to $-\pi / 2(\pi / 2)$ rad at which the sound pressure is double the sound pressure at $-\pi / 2(\pi / 2) \mathrm{rad}$.

Below (above) an angle of incidence of $-\theta_{m}\left(\theta_{m}\right) \mathrm{rad}$, the sound pressure decreases linearly as a function of the (cosine of the) angle of incidence, becoming, at an angle of incidence of $-\pi / 2$ $(\pi / 2) \mathrm{rad}$, half the sound pressure in the constant region.

Rindel's results are extended to finite rigid planes which are smaller in size compared to the wavelength by using results from Sivian and O'Neill (1932). Sivian and O'Neill make measurements at 13 different frequencies between $500 \mathrm{~Hz}$ and $13.5 \mathrm{kHz}$ on a disk with a diameter of $D=120 \mathrm{~mm}$ for angles of incidence from 0 to $\pi \mathrm{rad}$. For the lowest two frequencies, there are no angles of incidence at which the sound pressure is double the sound pressure at an angle of incidence of $\pi / 2 \mathrm{rad}$. For the eleven highest frequencies from 1 to $13.5 \mathrm{kHz}$ the value of $\theta_{m}$ rad is measured from their graphs. The value of $2 L$ is set equal to the average distance across the disk in the direction of measurement. This means that $2 L=D \pi / 4$. Sivian and O'Neill also make measurements on a $2 L=115 \mathrm{~mm}$ square plate at frequencies of 7 and $10 \mathrm{kHz}$ at angles of incidence from 0 to $\pi \mathrm{rad} . \theta_{m}$ rad also is measured from the two graphs of these experimental results.

The variable $y$ is set equal to $\pi / 2-\left|\theta_{m}\right|$ radians and the variable $x$ is set equal to $1 /(2 k L)$ where $k \mathrm{rad} / \mathrm{m}$ is the wave number of the sound. A linear regression of $\ln (y)$ against $\ln (x)$ is used to fit the relationship $y=b x^{m}$ to the experimental data set as shown in figure 3 . The value of $m$ is 0.505 with $95 \%$ confidence limits of \pm 0.023 and the value of $b$ is 1.78 with $95 \%$ confidence limits of \pm 0.17 . The value of $r^{2}$ is 0.973 . Since $\sqrt{\pi}=1.77$, these experimental results suggest that $m$ is equal to 0.5 and $b$ is equal to $\sqrt{\pi}$. Since $\pi / 2-\left|\theta_{m}\right|$ is equal to the second order expansion of $\cos \left(\left|\theta_{m}\right|\right)$ about $\left|\theta_{m}\right|=\pi / 2$ radians, approximate and replace $\pi / 2-\left|\theta_{m}\right|$ with $\cos \left(\left|\theta_{m}\right|\right)$. 
Because $\cos \left(\left|\theta_{m}\right|\right)$ is symmetrical about $\theta_{m}=0$, and $\cos \left(\left|\theta_{m}\right|\right)=\cos \left(\theta_{m}\right)$, this gives the limiting angle $\theta_{m}$ below which the sound pressure does not vary with angle of radiation (or incidence) as

$$
\theta_{m}=\left\{\begin{array}{cc}
0 & \text { if } k L \leq \frac{\pi}{2} \\
\arccos \left(\sqrt{\frac{\pi}{2 k L}}\right) & \text { if } k L>\frac{\pi}{2}
\end{array}\right.
$$

where $\theta_{m}$ is set equal to zero for values of $k L$ less than $\pi / 2$ so that it is defined sensibly for all values of $k L$. The regression is repeated with the variable $y$ set equal to $\cos \left(\theta_{m}\right)$. In this case, the value of $m$ is 0.485 with $95 \%$ confidence limits of \pm 0.022 and the value of $b$ is 1.60 with $95 \%$ confidence limits of \pm 0.15 . The value of $r^{2}$ is 0.973 .

It should be noted that $\phi_{l}$ (see equation (12)) is equal to $\theta_{m}$ for a strip of length $2 L$. Thus both $\phi_{l}$ and $\theta_{m}$ indicate the angle of incidence or radiation at which the finite size of the panel starts to have an effect. Also note that both $\phi_{l}$ and $\theta_{m}$ have the correct limit of $\pi / 2 \mathrm{rad}$ for a rigid infinite plane.

\section{Low Frequencies}

For sound incident at a grazing incident angle, the sound pressure is equal to the sound pressure of the incident sound pressure wave. Note that this is different from the grazing incidence sensitivity of a condenser microphone which decreases at high frequencies because of the variation in the phase of the incident sound pressure wave across the microphone diaphragm once the diaphragm becomes comparable to or greater than the wavelength. Thus the average sound pressure level due a grazing incident sound wave of unit sound pressure is set to be unity.

$$
p\left(\frac{\pi}{2}\right)=1 .
$$

The low frequency theory is based on the work of Muller, Black and Davis (1937) and Brüel and Rasmussen (1959). If a plane wave is incident normally on the end of an infinite rigid 
half cylinder of diameter $D \mathrm{~m}$, the sound pressure level (SPL) at the centre of the end of the finite half cylinder reaches a maximum of $10 \mathrm{~dB}$ above the SPL of the incident sound wave as the frequency is increased. The SPL at the centre of the end of the infinite half cylinder then proceeds to oscillate between 0 and $10 \mathrm{~dB}$ above the SPL of the incident sound wave as the frequency is increased further. However when the sound pressure increase all over the end of the finite half cylinder is multiplied by the sensitivity of an equivalent condenser microphone diaphragm at that radial position and averaged, the net increase in SPL at and above the first maximum is between 3 and $9 \mathrm{~dB}$. Thus the assumption of a constant $6 \mathrm{~dB}$ increase in sound pressure level above the frequency when the increase first becomes $6 \mathrm{~dB}$ is accurate to within 3 $\mathrm{dB}$.

The sound pressure level increase for normally incident sound waves on the diaphragm of a condenser microphone of diameter $D$ without grid, first reaches $6 \mathrm{~dB}$ for a value of $k L$ of 1.33 where $2 L=D \pi / 4$. Although this somewhat less than the value of $k L=\pi / 2=1.57$ for which $\theta_{m}$ becomes equal to zero, the average sound pressure $p_{0}$ on a panel of average dimensions $2 L$ by $2 W$ due to a normally incident sound pressure wave of unit sound pressure is approximated using

$$
p(0)=1+p_{W} p_{L},
$$

where

$$
p_{W}=\left\{\begin{array}{cc}
\sin (k W) & \text { if } k W \leq \frac{\pi}{2} \\
1 & \text { if } k W>\frac{\pi}{2}
\end{array}\right.
$$

and 


$$
p_{L}=\left\{\begin{array}{cc}
\sin (k L) & \text { if } k L \leq \frac{\pi}{2} \\
1 & \text { if } k L>\frac{\pi}{2}
\end{array} .\right.
$$

These equations are compared with the sound pressure increase for normally incident sound on the diaphragm of a one inch condenser microphone without grid in figure 4.

\section{Sound Pressure Increase}

The average ratio over the baffle of the sound pressure to the incident sound pressure for angles of incidence between $-\pi / 2(\pi / 2)$ rad and $-\theta_{m}\left(\theta_{m}\right)$ rad is estimated by linear interpolation of the sound pressure ratio as a function of the (cosine of the) angle of incidence between 1 at $-\pi / 2$ $(\pi / 2) \mathrm{rad}$ and $\mathrm{p}(0)$ at $-\theta_{m}\left(\theta_{m}\right) \mathrm{rad}$.

The average sound pressure on a finite rigid plane due to a plane sound wave of unity amplitude which is incident at an angle of $\theta$ radians relative to the normal to the plane is given by

$$
p(\theta)=\left\{\begin{array}{cc}
p(0) & \text { if } \cos (\theta) \geq \cos \left(\theta_{m}\right) \\
\frac{p(0) \cos (\theta)+p\left(\frac{\pi}{2}\right)\left(\cos \left(\theta_{m}\right)-\cos (\theta)\right)}{\cos \left(\theta_{m}\right)} & \text { if } \cos \left(\theta_{m}\right)>\cos (\theta) \geq 0
\end{array}\right.
$$

where interpolation in the cosine domain is used.

By reciprocity, this equation also gives the sound pressure radiated by a point source on a finite rigid plane at an angle of $\theta$ radians relative to the normal to the plane compared to the sound radiated in the absence of the plane. The value is averaged over all positions on the finite rigid plane. In Davy (2007), the interpolation in equation (20) is performed in the angle of incidence domain. In this paper and Davy (2008), the cosine of the angle of incidence is used. It should be noted that although this section has discussed rigid panels, the method is also used for openings. 


\section{Directivity of the sound radiation}

The relative sound pressure level $L(\theta)$ in the direction $\theta$ is

$$
L(\theta)=10 \log _{10}\left(\left|p_{T}(\theta)\right|^{2} p^{2}(\theta)\right)-10 \log _{10}\left(\left|p_{T}(0)\right|^{2} p^{2}(0)\right)
$$

If the transmission is into the shadow zone, that is $\frac{\pi}{2}<|\theta| \leq \pi$, then the above calculations are carried out for $\theta=\frac{\pi}{2}$ and the product $\left|p_{\operatorname{Trms}}\left(\frac{\pi}{2}\right)\right|^{2} p^{2}\left(\frac{\pi}{2}\right)$ in equation (21) is multiplied by the following diffraction correction.

$$
D(\theta)=\frac{1}{1-k z \cos (\theta)}
$$

where

$$
z=\frac{1}{\frac{1}{L}+\frac{1}{W}}
$$

Fluctuations in the shadow zone can be large. Equations (22) and (23) approximate the average trend of the experimental data (Sutton, 1990, Neish, 1997, Dewhirst, 2002, Li, 2005, and Potente et al., 2006).

In practical situations, scattering from turbulence and other objects places a lower limit on the relative sound pressure level. Let $L_{\max }$ be the maximum value of $L(\theta)$. It is assumed that the scattered sound level is $L_{S} \mathrm{~dB}$ below $L_{\text {max }}$. The predicted observed relative sound pressure level $L_{O}(\theta)$ is

$$
L_{O}(\theta)=10 \log _{10}\left(10^{L(\theta) / 10}+10^{\left(L_{\max }-L_{S}\right) / 10}\right) .
$$


$L_{S}$ is usually expected to be greater than $20 \mathrm{~dB}$. To predict the shadow zone data in Stead (2001), a value of $L_{S}$ equals $22 \mathrm{~dB}$ is used.

\section{COMPARISON WITH EXPERIMENTAL RESULTS FOR DUCTS}

The theory developed in this paper is primarily two dimensional. If the dimensions of the opening, panel or baffle are not constant parallel to and perpendicular to the plane in which the directivity is measured, it is necessary to calculate an average dimension in the two directions. In this paper, for a circular opening, panel or baffle, the length and width are set equal to the $\pi / 4$ times the diameter. The theoretical results for ducts given in this section are computed using a wall absorption coefficient of 0.05 .

Most of the initial comparisons between the experimental results and the theory described in this paper were for the case of openings and panels mounted in the wall of a room where the sound source was external to the room and the microphone was inside the room. In these experiments the sound source was always rotated so that its direction of maximum sound radiation was directed towards the opening or the panel. In this situation, the directivity of the sound source had no effect on the results. The microphone in the room was relatively omnidirectional and thus its directivity also did not need to be considered. Thus there was no need to use equation (14).

However, when the directivity of the sound radiated from the opening at the end of a duct was considered, it became apparent that the directivity of the sound source at the other end of the duct needed to be included and equation (14) was introduced into the theoretical model. It was initially thought that the $2 r$ in equation (14) should be set equal to the diameter of the sound source if this value was known. However it was observed that this did not always produce the best agreement between theory and experiment. 
For each duct and each frequency, $2 r$ was set equal to value that made the average value over the angle of radiation of the difference between experiment and theory equal to zero or as close to zero as possible. These values of $2 r$ varied over a wide range. However, somewhat surprisingly, it was observed that the values of $2 r$ tended to decrease with increasing frequency. The average value of $2 r$ over a large number of experimental results was approximately the wavelength $\lambda$ of sound in air. This made the average value of $k r$ approximately equal to $\pi$.

One of the reasons for this strange result is that smaller sound sources have to be used as the frequency is increased in order to achieve constant sound power output. Secondly as the frequency increases, loudspeakers only radiate efficiently from a decreasing area around the centre of their cones because of wave motion in their cones. Thus the physics tends to require the use of constant $k r$ sound sources.

The range of the $k r$ values was investigated further by setting $k r$ for each duct equal to value that made the average value over all angles of radiation and all frequencies of the differences between experiment and theory equal to zero. The standard deviation over all angles of radiation and all frequencies of the differences between experiment and theory was determined as an estimate of the goodness of agreement between theory and experiment.

Table I shows the values of $k r$ and the standard deviations for 10 outdoor measurements on circular ducts with diameters ranging from 305 to $1220 \mathrm{~mm}$ (Neish 1997, Potente et al. 2006). The values of $k r$ range from 1.55 to 3.3. The standard deviations range from 1.7 to $4.6 \mathrm{~dB}$. An example of the results used to calculate Table I is shown in figure 5.

Table II shows the values of $k r$ and the standard deviations for 18 anechoic measurements on ducts with cross sectional dimensions ranging from 40 to $240 \mathrm{~mm}$ (Croft 1979, Sutton 1990, Dewhirst 2002, Li 2005). The values of $k r$ range from 2.75 to 13 for ducts driven with third 
octave bands of random noise which are unflanged except for the thickness of their wall sound insulation. The flanged duct has a $k r$ of 20 and the duct driven with a pure tone has a $k r$ of 30. The standard deviations range from 1.1 to $8.0 \mathrm{~dB}$. The three biggest standard deviations were for three of Li's four measurements. Li measured at angles of one degree increments. His measurements may have picked up more deviations than the other coarser angular measurements. An example of the results used to calculate Table II is shown in figure 6.

\section{COMPARISON WITH EXPERIMENTAL RESULTS FOR ROOMS}

For the scale model anechoic room and near field acoustical holography measurements considered in this section, the procedure described at the start of the previous section was repeated. The average value of $k r$ was 1.78 and this value is used for the theoretical calculations in this section.

Oldham and Shen (1982), Shen and Oldham (1982) and Oldham and Shen (1983) conduct scale model investigations of the sound radiation from an opening or panel mounted in the wall of a room. They use a box with external dimensions of $0.5 \times 0.5 \times 0.5 \mathrm{~m}$ which they rotate in an anechoic room. The internal dimensions of their box are approximately $0.3 \times 0.3 \times 0.3 \mathrm{~m}$, but "two of the inner walls were inclined in order to improve the diffusion of the sound field. Four piezo-electric tweeters having a frequency range extending to $40 \mathrm{kHz}$ were placed in the four lower corners of the model room with their axes inclined to the walls. 1/3 octave band filtered white or pink noise was supplied to the loudspeakers to produce a reverberant field inside the model room. Spherical diffusers of diameter about $4 \mathrm{~cm}$ were hung from the ceiling in order to improve the diffusion of the sound field." No reverberation times are given, so the absorption coefficient of the internal walls of the box is assumed to be 0.05 for the theoretical calculations in this section. 
Table III shows the averages and standard deviations over all angles of radiation and all frequencies of the differences between the experimental directivity and the theoretical directivity.The first length of the two aperture dimensions is the length of the opening or panel in the plane of measurement. Oldham and Shen (1982) observe that their results for an opening depended mainly on the value of the product of the frequency with the length of the opening in the plane of measurement. They average all of their results which have the same values of this product. The difference between their average results and the theoretical results are also shown in Table III. An example of the results used to calculate Table III is shown in figure 7.

The general trend is for both the magnitudes of the average differences and the standard deviations of the differences to become greater as the panel thickness increases and decreases the critical frequency. To obtain theoretical values at the coincidence peaks which are in reasonable agreement with the experimental peaks, damping loss factors of 0.23 for plexiglass and 0.1 for aluminium are assumed. These values are about one hundred times greater than the typical values of 0.002 and 0.001 . Also the value of the damping loss factor used for the concrete panel is 0.01 which is at the upper end of the expected in situ range.

The problem appears to be the inadequacy of equation (13) to properly model the wave impedance of a finite panel in the vicinity of and above the critical frequency of the panel. There have been some suggestions of this in previous comparisons made by the author on thin panels whose critical frequencies are near the high frequency end of the measured frequency range. It is much more obvious for a thick panel whose critical frequency is near the low frequency end of the measured frequency range.

Equation (13) is only strictly valid for the forced wave in an infinite panel. Ljunggren (1991) has pointed out that one of the problems with equation (13), when it is applied to a finite 
panel, is the spatial rise length. It can take a considerable distance from the edge of the panel for the forced bending wave to reach the velocity implied by equation (13). It is possible for this distance to be much greater than the dimensions of the panel. A second problem is that the waves reflected from the edges of the panel propagate with the free bending wavelength rather than the trace wavelength forced by the airborne sound. An attempt was made to model the vibration of the $5 \mathrm{~mm}$ thick concrete panel as the forced wave in an infinite limp panel and the rest of the vibration as free bending waves. Unfortunately this approach made the disagreement worse. It produced an average difference of $-2.6 \mathrm{~dB}$ and a standard deviation of $8.3 \mathrm{~dB}$.

Rowell and Oldham (1996) used near field acoustical holography to measure the directivity of the sound insulation of homogeneous, profiled and composite panels for the octave band frequencies from $125 \mathrm{~Hz}$ to $4 \mathrm{kHz}$. They also gave the results of far field measurements on a 6 $\mathrm{mm}$ thick aluminium panel measuring $2400 \times 1200 \mathrm{~mm}$ in one third octave frequency bands from 2 to $6.3 \mathrm{kHz}$. The averages and standard deviations of the differences between their experimental results and the theoretical results over angles and frequencies are shown in Table IV. An example of the results used to calculate Table IV is shown in figure 8. A damping loss factor of 0.23 is used for the $6 \mathrm{~mm}$ thick aluminium panels. The use of such a large loss factor is required because the experimental coincidence peaks are not as large as expected and tend to disappear altogether at higher frequencies. This is discussed by Rowell and Oldham (1996).

The profiled panels measured are a quasi-sinusoidally corrugated $0.5 \mathrm{~mm}$ thick steel panel and three trapezoidally corrugated $0.7 \mathrm{~mm}$ thick steel panels. The directivity of the sound insulation was measured in both a plane at right angles to the corrugations and in a plane parallel to the corrugations. The damping loss factor used for all the theoretical calculations on the profiled panels is 0.0004 which is in the normal range for steel. The magnitudes of the averages 
and the standard deviations in a plane parallel to the corrugations were equal to or larger than those in a plane at right angles to the corrugations. This is believed to be due to the much lower critical frequency parallel to the corrugations and the problems with equation (13) at and above the critical frequency.

The composite panels consisted of a layer of thermally insulating polyurethane material sandwiched between and bonded to two slightly profiled steel panels. The directivity of the sound insulation was measured in two planes at right angles to each other. The damping loss factor used for the theoretical calculations on the composite panels was 0.008 . The critical frequencies used for the two panels were 500 and $700 \mathrm{~Hz}$ respectively.

The mean and the root mean square of the differences between experiment and theory for the 55 sets of data summarised in Tables I to IV are 0.2 and $4.4 \mathrm{~dB}$. Thus the theory does a reasonable job of predicting the average trend of the experimental data, but the variation of the experimental data about the theoretical data is large. It must be remembered that the experimental measurements are difficult to make accurately because of breakout noise through the walls of the duct or room and residual reflections from other objects. It should also be noted that the $4.4 \mathrm{~dB}$ is relatively small compared with the possible $60 \mathrm{~dB}$ range of values from +20 to $-40 \mathrm{~dB}$. Nevertheless, it is believed that equation (13) does not adequately model the impedance of a finite panel in the vicinity of and above the critical frequency.

\section{CONCLUSION}

For a panel or opening excited by sound which is incident from the opposite side, the model presented in this paper can be used to predict the sound pressure level radiated at a particular angle to the normal of the panel or opening, relative to the sound pressure level radiated in the direction of the normal. The model includes a theory to predict the angular 
distribution of the incident sound based on the sound absorption coefficient of the walls of the room or the duct. A line source directivity model is also used when the sound source is inside the room or duct. On average, the length of this line source model has been experimentally determined to be the wavelength of the sound for loudspeaker sound sources in a duct. For a sound source in a room $k r$ is set equal to 1.78 where $2 r$ is the length of the line source model.

The model also includes an empirical method for predicting the diffraction effect of the finite size of the baffle in which the panel or opening is mounted as a function of angle of radiation or incidence up to $90^{\circ}$ to the normal. Another empirical diffraction model is used for radiation into or diffraction from the shadow zone where the angle of radiation or incidence is greater than ninety degrees to the normal.

The model is developed for the transmission of sound from a room wall or duct end via a panel or opening into a free field space. However, by the principle of reciprocity, it can also be used for transmission in the other direction. In some situations it is necessary to consider the effects of scattering from atmospheric turbulence and other objects and the effects of noise which breaks out through the duct or room walls.

The average difference between experiment and theory is only $0.2 \mathrm{~dB}$. However, the experimental results show significant variability about the theoretical predictions. The root mean square of the differences between experiment and theory is $4.4 \mathrm{~dB}$. The differences will be greater when the theory is compared with other experimental data because the theoretical sound source directivity used in this paper has been adjusted to minimise the differences. The agreement is worse when the critical frequency of the panel decreases as the thickness of the panel increases. This is believed to be due to the inadequacy of the model for the wave impedance of a panel at and above the critical frequency of the panel. It should be noted that the 
experimental difficulties also increase as the sound insulation of the panel increases with increasing thickness.

\section{ACKNOWLEDGEMENTS}

Doug Growcott suggested the directivity problem considered in this paper as a suitable topic for research because of the paucity of information available in the literature for use by acoustical consultants. Matthew Stead, Jens Rindel, Colin Hansen, Xun Li, David Luck, and Athol Day provided experimental data and reports to the author. The experimental data was often in machine readable form. Vlad Pavasovic, Thomas Kannanaikkel John, and Kai Fisher worked on this research problem while students of the author.

\section{REFERENCES}

Allen, C. H. (1960). "Noise control in ventilation systems," in Noise Reduction edited by Beranek, L. L., (McGraw-Hill, New York).

Beranek, L. L. (1954). Acoustics, (Republished by the American Institute of Physics, New York, 1986).

Bies, D.A., and Hansen, C.H., (2003). Engineering Noise Control: Theory and Practice, Third Edition (Spon Press, London and New York).

Björk, E. A. (1994). "Experimental study of measures to reduce noise radiated from powerstation exhaust stacks," Noise Control Eng. J. 42, 171-178.

Brüel, P. V., and Rasmussen, G. (1959). "Free field response of condenser of condenser microphones”, Brüel and Kjcer Technical Review, 12-17, Part 1; 1-15, Part 2.

Cremer, L., and Heckl, M. (1973). Structure-Borne Sound, (Springer-Verlag Company, New York). 
Croft, G. J. (1979). Noise Directivity of Exhaust Stacks, (Final year thesis for the Honours Degree of Bachelor of Engineering, University of Adelaide, Adelaide, Australia). This thesis has been lost and Croft's results only exist as a graph in Dewhirst (2002).

Davy, J. L. (2004). "The radiation efficiency of finite size flat panels," Gold Coast, November 35, Acoustics 2004, Transportation Noise and Vibration - The New Millennium, Proceedings of the Annual Conference of the Australian Acoustical Society, edited by Mee, M. J., Hooker, R. J. and Hillock, I. D. M., (Book ISBN 909882-21-5, CDROM ISBN 0-909882-22-3 ISSN 14460998, published by Australian Acoustical Society, Castlemaine, Victoria, Australia), pages 555560.

Davy, J. L. (2007). "A model for predicting diffraction on a finite flat surface as a function of angle of incidence and surface size," on the CD-ROM: Istanbul, August 28-31, Global Approaches to Noise Control, Proceedings of Inter-noise 2007, the 36th International Congress and Exhibition on Noise Control Engineering, (published by the Turkish Acoustical Society), paper 21.

Davy, J. L. (2008). "The directivity of the forced radiation of sound from panels and openings including the shadow zone", on the DVD: Paris, 29 June-4 July, Proceedings of Acoustics'08, ISBN 978-2-9521105-4-9, pages 3833-3838, paper 11. Abstract, J. Acoust. Soc. of Am. 123 (5 Part 2):3499 ISSN 0001 4966, Acta Acustica united with Acustica, 94(Supplement 1):S531 ISSN 1610-1928.

Davy, J. L., and Kannanaikkel John, T. (2006). "The angular distribution of sound incident on a panel or opening," J. Acoust. Soc. of Am. 120, 3187(A).

Davy, J. L., and Kannanaikkel John, T. (2007). "The directivity of the forced radiation of sound from panels and openings," on the CDROM: Cairns, July 9-12, Proceedings of the Fourteenth 
International Congress on Sound and Vibration, ICSV14, edited Randall, B. (ISBN 97807334 $25165)$, paper 7.

Davy, J. L., and Pavasovic, V. (2006). "The directivity of sound radiated from a panel or opening excited by sound incident from the other side," Christchurch, November 20-22, Proceedings of Acoustics 2006, Noise of Progress, First Australasian Acoustical Societies' Conference, edited by McMinn, T., (ISBN: 978-0-909882-25-9, ISSN: 1446-0998), pages 133-140.

Dewhirst, M. (2002). Exhaust Stack Directivity, (Final report, Final year design project for the Degree of Bachelor of Engineering, University of Adelaide, Adelaide, Australia).

Fisher, K. R. (2006). Directivity of the sound transmission through finite sized apertures and panels (Bachelor of Applied Science (Physics) Honours dissertation, RMIT University, Melbourne, Australia), Available from K. R. Fisher at Arup Acoustics, Melbourne, Australia or the author of this paper.

Joseph, P., and Morfey, C. L. (1999). "Multimode radiation from an unflanged, semi-infinite circular duct," J. Acoust. Soc. of Am. 105, 2590-2600.

Levine, H., and Schwinger, J. (1948). "On the radiation of sound from an unflanged circular pipe," Phys. Rev. 73, 383-406.

Li, X. (2005). Milestone report for determination of the duct directivity in an anechoic chamber, (University of Adelaide, Adelaide, Australia).

Ljunggren, S., (1991). "Airborne sound insulation of thin walls," J. Acoust. Soc. of Am. 89, 2324-2337.

Muller, G. G., Black, R., and Davis, T. E. (1937). "The diffraction produced by cylindrical and cubical obstacles and by circular and square plates," J. Acoust. Soc. of Am. 10, 6-13. 
Neish, M. J. (1997). Predicting sound directivity at a ventilation duct termination, (Final year project, School of Mechanical Engineering, The University of Technology, Sydney, Australia). Oldham, D. J., and Shen, Y. (1982). "A scale model investigation of sound radiation from a large aperture in a building," J. Applied Acoustics 15, 397-350.

Oldham, D. J., and Shen, Y. (1983). "A scale model investigation of sound radiation from building elements," J. Sound and Vib. 84, 331-350.

Pavasovic, V. (2006). The radiation of sound from surfaces at grazing angles of incidence (Master of Applied Science dissertation, RMIT University, Melbourne, Australia), available for download from the Australian Digital Theses Program http://adt.lib.rmit.edu.au/adt/public/adtVIT20060911.115939, last viewed on 22 September 2009.

Potente, D., Gauld, S., and Day, A. (2006). "Directivity loss at a duct termination," Christchurch, November 20-22, Proceedings of Acoustics 2006, Noise of Progress, First Australasian Acoustical Societies' Conference, edited by McMinn, T., (ISBN: 978-0-909882-259, ISSN: 1446-0998), pages 283-289.

Rindel, J. H. (1975). Transmission of traffic noise through windows - Influence of incident angle on sound insulation in theory and experiment, Report No. 9, The Acoustics Laboratory, Technical University of Denmark, Lyngby, Denmark.

Roberts, J. (1983). "The prediction of directional sound fields," Transactions of the Institution of Engineers, Australia, Mechanical Engineering, Paper M1153, ME8(1):16-22, ISSN 0727-7369.

Rowell, M. A., and Oldham, D. J. (1996). "Determination of the directivity of a planar noise source by means of near field acoustical holography, Part III: Measurements on homogeneous, profiled and composite panels," J. Sound and Vib. 180, 99-118.

Sharland, I. (1972). Woods practical guide to noise control, (Woods of Colchester, Colchester). 
Shen, Y., and Oldham, D. J. (1982). "Sound radiation from building elements," J. Sound and Vib. 84, 11-33.

Sivian, L. J., and O’Neil, H. T. (1932). “On sound diffraction caused by rigid circular plate, square plate and semi-finite screen”, J. Acoust. Soc. of Am. 3, 483-510.

Stead, M. (2001). Sound Reduction for Reverberant, Direct and Diffracted Sound through Single Isotropic Glass Panels of Finite Size, (Master of Engineering Science dissertation, Department of Mechanical Engineering, Monash University, Melbourne, Australia).

Sutton, M. (1990). Noise Directivity of Exhaust Stacks, (Final year thesis for the Honours Degree of Bachelor of Engineering, University of Adelaide, Adelaide, Australia).

Watters, B. G., Labate, S., and Beranek, L. L. (1955). “Acoustical behaviour of some engine test cell structures,” J. Acoust. Soc. of Am. 27, 449-456.

Wells, R. J., and Crocker, B. E. (1953). "Sound radiation patterns of gas turbine exhaust stacks," J. Acoust. Soc. of Am. 25, 433-437.

Woods, R. I. (1972). Noise control in mechanical services, (Sound Attenuaters Ltd and Sound Research Laboratories Ltd, Colchester). 
TABLE I. Values of $k r$ and standard deviations over all angles of radiation and all frequencies of the differences between the experimental directivity ((a) Neish, 1997, and (b) Potente et al., 2006) and the theoretical directivity for 10 outdoor measurements on circular ducts.

\begin{tabular}{|l|l|l|l|l|}
\hline $\begin{array}{l}\text { Diameter of duct } \\
(\mathrm{mm})\end{array}$ & $\begin{array}{l}\text { Length of duct } \\
(\mathrm{m})\end{array}$ & $\begin{array}{l}\text { Measurement } \\
\text { distance (m) }\end{array}$ & $k r$ & $\begin{array}{l}\text { Standard } \\
\text { deviation (dB) }\end{array}$ \\
\hline $305(\mathrm{~b})$ & 3 & 1 & 3.3 & 2.3 \\
\hline $305(\mathrm{~b})$ & 3 & 3 & 2.1 & 2.3 \\
\hline $400(\mathrm{a})$ & 8 & 2 & 1.55 & 1.7 \\
\hline $610(\mathrm{~b})$ & 3 & 2 & 3.25 & 2.5 \\
\hline $610(\mathrm{~b})$ & 6 & 4 & 2.6 & 3.6 \\
\hline $914(\mathrm{~b})$ & 4.8 & 3 & 2.3 & 4.5 \\
\hline $914(\mathrm{~b})$ & 7.8 & 3 & 1.8 & 3.6 \\
\hline $914(\mathrm{~b})$ & 7.8 & 6 & 2.65 & 3.3 \\
\hline $1220(\mathrm{a})$ & 12 & 3 & 1.55 & 2.8 \\
\hline $1220(\mathrm{a})$ & 12 & 6 & 2.2 & 2.4 \\
\hline
\end{tabular}


TABLE II. Values of $k r$ and standard deviations over all angles of radiation and all frequencies of the differences between the experimental directivity ((a) Croft, 1979, (b) Sutton, 1990, (c) Dewhirst, 2002, and (d) Li, 2005) and the theoretical directivity for 18 anechoic room measurements on ducts.

\begin{tabular}{|l|l|l|}
\hline Duct cross section (mm) & $k r$ & Standard deviation (dB) \\
\hline 85 diameter (b) & 4.1 & 2.9 \\
\hline 85 diameter (b) & 5 & 3.1 \\
\hline $80 \times 80(\mathrm{~b})$ & 3.7 & 2.8 \\
\hline $120 \times 40(\mathrm{~b})$ & 2.75 & 2.7 \\
\hline $40 \times 120(\mathrm{~b})$ & 4.6 & 1.7 \\
\hline $80 \times 40(\mathrm{~b})$ & 4.1 & 2.9 \\
\hline $40 \times 80$ (b) & 6 & 3.0 \\
\hline 85 diameter pure tone excitation (b) & 30 & 4.3 \\
\hline 112 diameter (a) & 3.37 & 1.1 \\
\hline $120 \times 120$ (c) & 8 & 2.9 \\
\hline $80 \times 160$ (c) & 8 & 2.5 \\
\hline $160 \times 80$ (c) & 3.2 & 3.1 \\
\hline $80 \times 240$ (c) & 16 & 3.2 \\
\hline $240 \times 80$ (c) & 3.05 & 3.9 \\
\hline 130 diameter 260 diameter flange (d) & 20 & 3.1 \\
\hline 130 diameter (d) & 13 & \\
\hline $80 \times 160(\mathrm{~d})$ & 2.8 & \\
\hline $160 \times 80(\mathrm{~d})$ & & \\
\hline
\end{tabular}


TABLE III. The averages and standard deviations over all angles of radiation and all frequencies of the differences between the experimental directivity ((a) Oldham and Shen, 1982, (b) Shen and Oldham, 1982, and (c) Oldham and Shen, 1983) and the theoretical directivity for 12 anechoic room measurements on openings and panels in the wall of a model room.

\begin{tabular}{|l|l|l|l|l|}
\hline $\begin{array}{l}\text { Material \& } \\
\text { Thickness (mm) }\end{array}$ & Size (mm) & $\begin{array}{l}\text { Damping } \\
\text { factor }\end{array}$ & $\begin{array}{l}\text { Average } \\
\text { difference (dB) }\end{array}$ & $\begin{array}{l}\text { Standard } \\
\text { deviation (dB) }\end{array}$ \\
\hline Opening (a) & $100 \times 50$ & & -0.1 & 1.6 \\
\hline Opening (a) & $100 \times 100$ & & 0.7 & 2.2 \\
\hline Opening (a) & $50 \times 100$ & & -1.6 & 2.3 \\
\hline Opening (a) & Average & & 0.8 & 1.4 \\
\hline Concrete 5 (b) & $200 \times 150$ & 0.01 & 1.0 & 6.2 \\
\hline Aluminium 0.5 (b) & $100 \times 50$ & 0.1 & 1.5 & 2.0 \\
\hline Plexiglass 1 (c) & $300 \times 100$ & 0.23 & 0.4 & 1.6 \\
\hline Plexiglass 2 (c) & $200 \times 200$ & 0.23 & 0.4 & 4.0 \\
\hline Aluminium 0.5 (c) & $100 \times 50$ & 0.1 & 1.3 & 2.6 \\
\hline Plexiglass 6 (c) & $100 \times 300$ & 0.23 & 3.0 & 6.0 \\
\hline Plexiglass 6 (c) & $300 \times 100$ & 0.23 & 4.1 & 7.3 \\
\hline Plexiglass 8 (c) & $300 \times 200$ & 0.23 & 5.3 & 7.4 \\
\hline
\end{tabular}


TABLE IV. The averages and standard deviations over all angles of radiation and all frequencies of the differences between the experimental directivity (Rowell and Oldham, 1996) and the theoretical directivity for 14 near field acoustical holography measurements on panels in the wall of a room. The results for the third aluminium panel are from far field measurements.

\begin{tabular}{|l|l|l|l|l|l|}
\hline $\begin{array}{l}\text { Material } \\
\text { Thickness }(\mathrm{mm})\end{array}$ & Size $(\mathrm{mm})$ & Corrugation & $\begin{array}{l}\text { Damping } \\
\text { loss } \\
\text { Direction }\end{array}$ & $\begin{array}{l}\text { Average } \\
\text { difference } \\
(\mathrm{dB})\end{array}$ & $\begin{array}{l}\text { Standard } \\
\text { deviation } \\
(\mathrm{dB})\end{array}$ \\
\hline Aluminium 6 & $920 \times 1200$ & & 0.23 & -1.0 & 2.6 \\
\hline Aluminium 6 & $1200 \times 920$ & & 0.23 & -0.6 & 3.6 \\
\hline Aluminium 6 & $2400 \times 1200$ & & 0.23 & -0.1 & 6.1 \\
\hline Steel 0.5 Across & $805 \times 1200$ & Quasi-sinusoidal & 0.0004 & 0.3 & 3.5 \\
\hline Steel 0.5 Parallel & $1200 \times 805$ & Quasi-sinusoidal & 0.0004 & 0.3 & 4.7 \\
\hline Steel 0.7 Across & $920 \times 1200$ & Trapezoidal & 0.0004 & 0.8 & 4.1 \\
\hline Steel 0.7 Parallel & $1200 \times 920$ & Trapezoidal & 0.0004 & -2.2 & 7.1 \\
\hline Steel 0.7 Across & $920 \times 1200$ & Trapezoidal & 0.0004 & -0.5 & 4.7 \\
\hline Steel 0.7 Parallel & $1200 \times 920$ & Trapezoidal & 0.0004 & -0.8 & 6.3 \\
\hline Steel 0.7 Across & $882 \times 1200$ & Trapezoidal & 0.0004 & 0.2 & 3.1 \\
\hline Steel 0.7 Parallel & $1200 \times 882$ & Trapezoidal & 0.0004 & 2.3 & 8.6 \\
\hline Sandwich panel & $1200 \times 1200$ & & 0.008 & -0.9 & 5.7 \\
\hline Sandwich panel & $1200 \times 1200$ & & 0.008 & 0.3 & 5.2 \\
\hline Sandwich panel & $1200 \times 1200$ & & 0.008 & -1.8 & 4.8 \\
\hline Sandwich panel & $1200 \times 1200$ & & 0.008 & -2.5 & 4.5 \\
\hline
\end{tabular}




\section{FIGURE CAPTIONS}

Figure 1. Sound incident at an angle of $\phi$ to the normal to a panel or opening and radiated at an angle of $\theta$ to the normal.

Figure 2. Calculating the number of wall reflections before sound hits the panel or opening at an angle of $\phi$ to the normal.

Figure 3: Best fit of $y=\pi / 2-\left|\theta_{m}\right|$ rad versus $x=1 /(2 k L)$.

Figure 4: Comparison of the experimentally determined sound pressure increase for normally incident sound on the diaphragm of a one inch condenser microphone without grid (Brüel and Rasmussen, 1959) with the theory of equations (17), (18) and (19).

Figure 5: Experimental (Potente et al., 2006) and theoretical sound pressure level relative to that at $0^{\circ}$ for a $914 \mathrm{~mm}$ diameter $7800 \mathrm{~mm}$ long duct measured $3000 \mathrm{~mm}$ from the centre of the duct end opening at an angle of radiation relative to the normal of $105^{\circ}$ as a function of $k a$.

Figure 6: Experimental (Sutton, 1990) and theoretical sound pressure level for an $80 \mathrm{~mm}$ square cross section $750 \mathrm{~mm}$ long duct as a function of angle of radiation relative to the normal when $\mathrm{ka}$ $=11.6$.

Figure 7. Experimental (Oldham and Shen, 1983) and theoretical sound pressure level relative to that at $0^{\circ}$ for a $1 \mathrm{~mm}$ thick Plexiglas panel in the wall of a room as a function of angle of radiation relative to the normal when $k a=87$.

Figure 8. Experimental (Rowell and Oldham, 1996) and theoretical sound pressure level relative to that at $0^{\circ}$ for a quasi-sinusoidally corrugated $0.5 \mathrm{~mm}$ thick steel panel measured in a plane parallel to the corrugations at an angle of radiation relative to the normal of $90^{\circ}$ as a function of $k a$. 


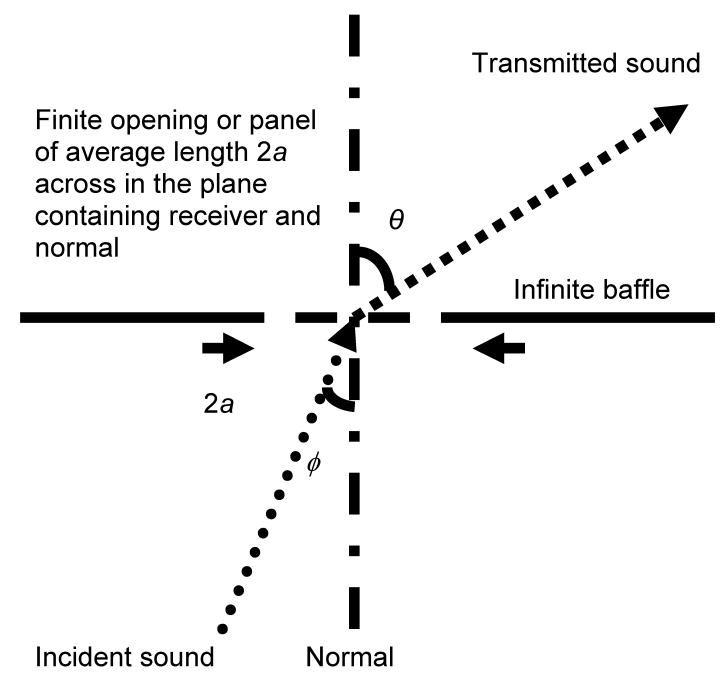




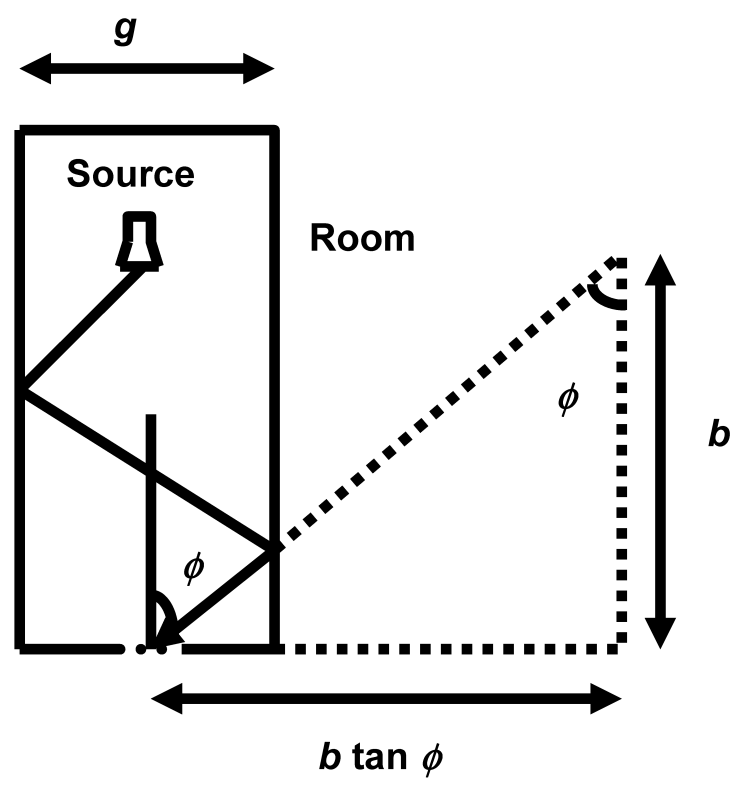




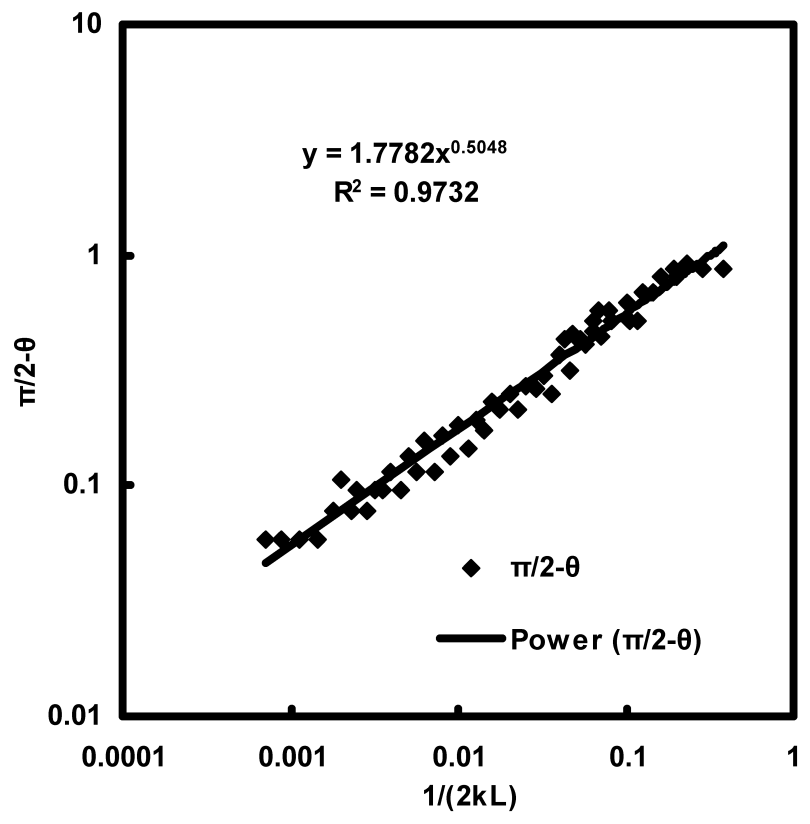




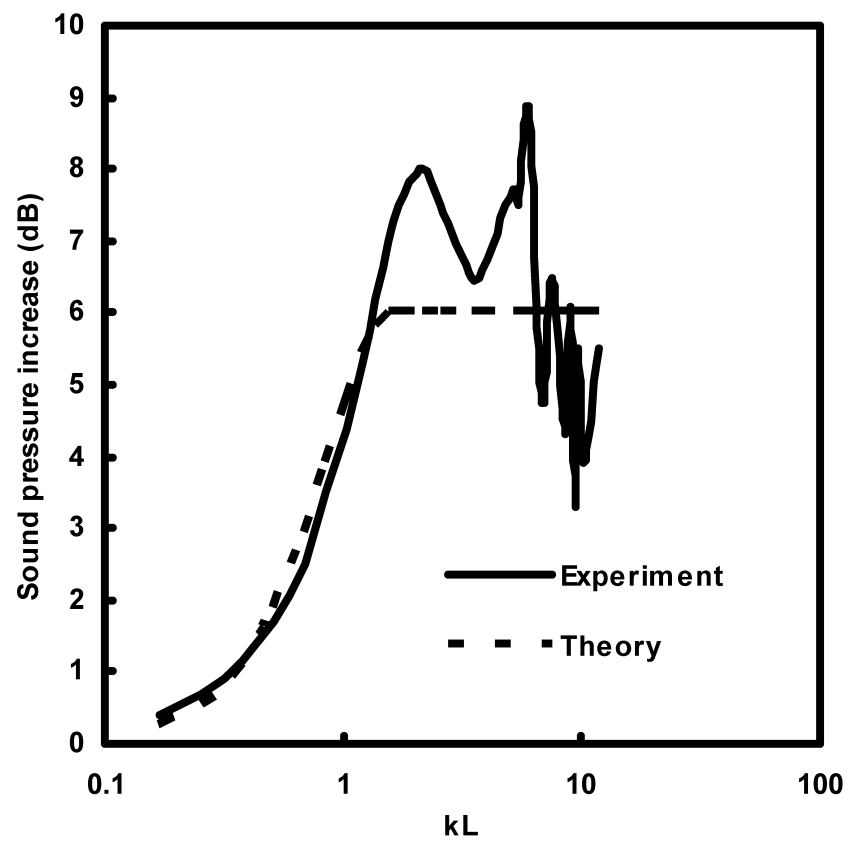




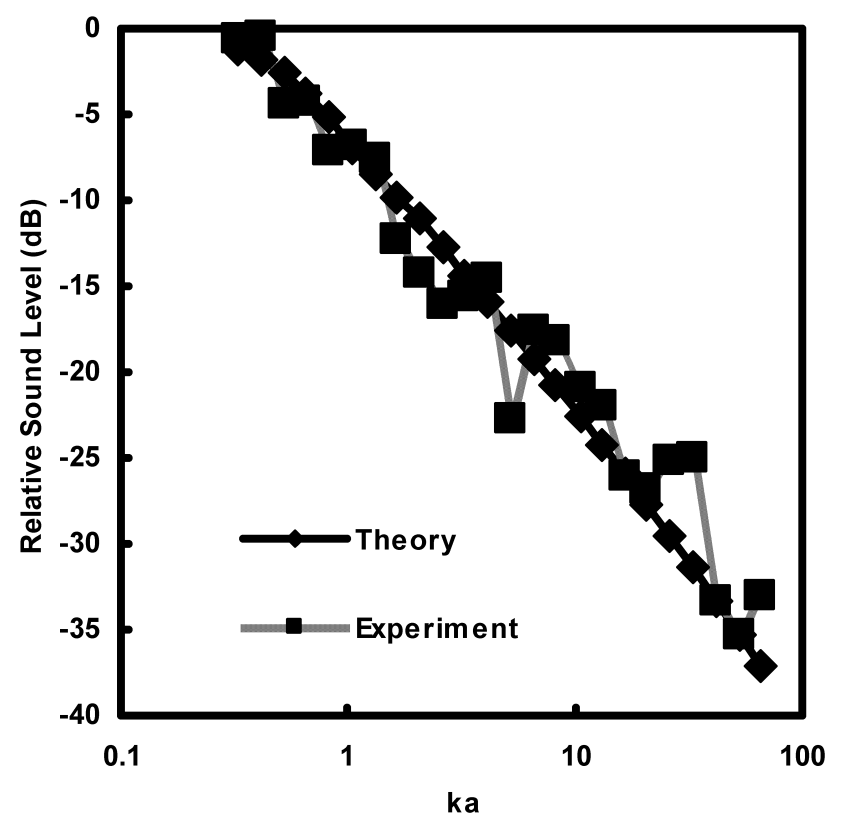




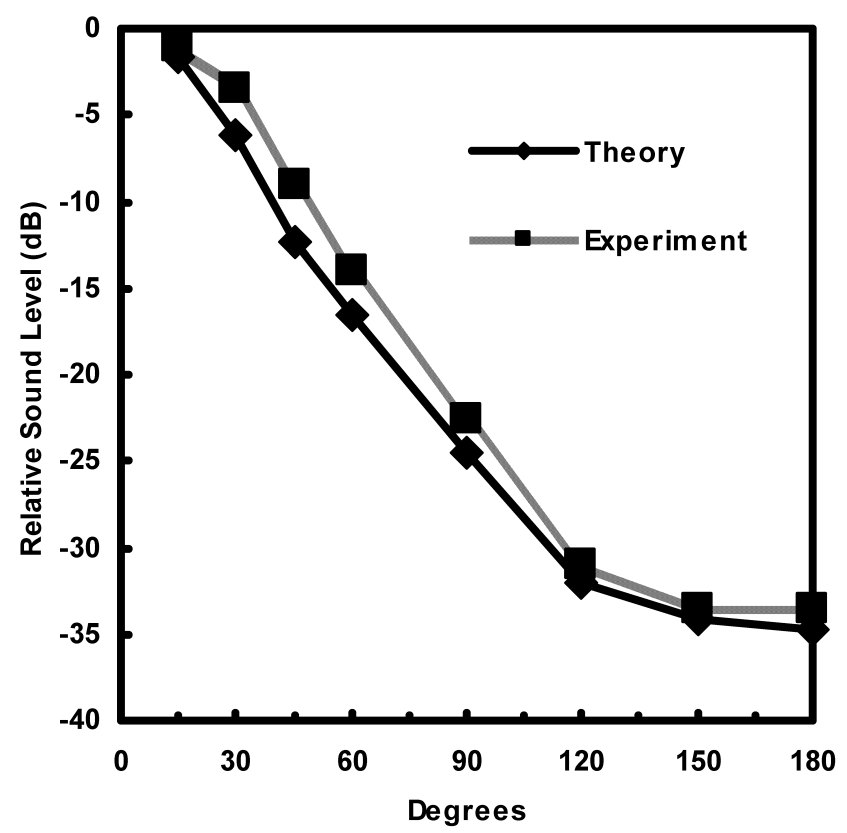




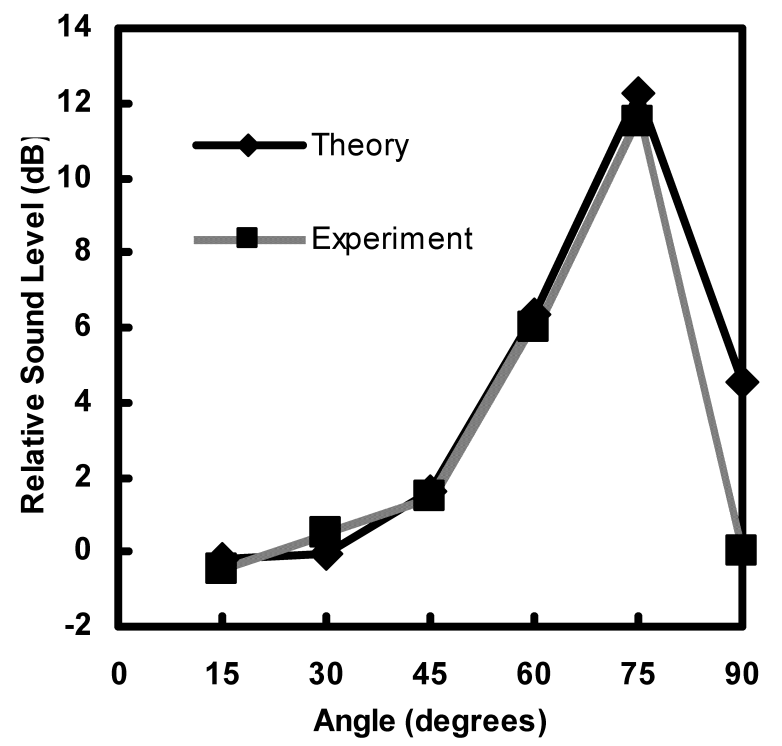




$$
4
$$

